\title{
Open Subsets in a Stein Space with Singularities
}

\author{
Jing Zhang
}

\begin{abstract}
Serre proved that a domain $Y$ in $\mathbb{C}^{n}$ is Stein if and only if $H^{i}\left(Y, \mathcal{O}_{Y}\right)=0$ for all $i>0$. Laufer showed that if $Y$ is an open subset of a Stein manifold of dimension $n$ and $H^{i}\left(Y, \mathcal{O}_{Y}\right)$ is a finite dimensional complex vector space for every $i>0$, then $Y$ is Stein. Vâjâitu generalized these theorems to singular Stein space of dimension $n$. In this paper, we consider singular Stein spaces $X$ with arbitrary dimension and give necessary and sufficient conditions for an open subset $Y$ in $X$ to be Stein. We show that if $Y$ is an open subset of a reduced Stein space $X$ with arbitrary dimension and singularities, then $Y$ is Stein if and only if $H^{i}\left(Y, \mathcal{O}_{Y}\right)$ is a finite dimensional complex vector space for every $i>0$. Without cohomology condition, if $X-Y$ is a closed subspace of $X$, then we show that the geometric condition of the boundary $X-Y$ determines the Steinness of $Y$. More precisely, we show that if $X$ is normal and the boundary $X-Y$ is the support of an effective $\mathbb{Q}$-Cartier divisor, or $X-Y$ is of pure codimension 1 and does not contain any singular points of $X$, then $Y$ is Stein.
\end{abstract}

\section{Introduction}

We work over the field $\mathbb{C}$ of complex numbers.

Let $X$ be a Hausdorff topological space. $\left(X, \mathcal{O}_{X}\right)$ is a complex space if every point of $X$ has a neighborhood $U$ such that $\left(U, \mathcal{O}_{U}\right)$ is isomorphic to a closed complex subspace $\left(A, \mathcal{O}_{A}\right)$ of a domain $D \subset \mathbb{C}^{m}$ for some $m \in \mathbb{N}$, where $A$ is the support of the analytic coherent $\mathcal{O}_{D}$ sheaf $\mathcal{O}_{A}=\mathcal{O}_{D} /\left.\mathcal{I}\right|_{A}$, and $\mathcal{I} \subset \mathcal{O}_{D}$ is an analytic coherent ideal sheaf.

A complex space $Y$ is Stein if it is both holomorphically convex and holomorphically separable [8, pp. 293-294, Theorem 63.2]. We say that $Y$ is holomorphically convex if for any discrete sequence $\left\{y_{n}\right\} \subset Y$, there is a holomorphic function $f$ on $Y$ such that the supremum of the set $\left\{\left|f\left(y_{n}\right)\right|\right\}$ is $\infty$. $Y$ is holomorphically separable if for every pair $x, y \in Y, x \neq y$, there is a holomorphic function $f$ on $Y$ such that $f(x) \neq f(y)$. By Cartan's Theorem B, a complex space $Y$ is Stein if and only if $H^{i}(Y, \mathcal{F})=0$ for every analytic coherent sheaf $\mathcal{F}$ on $Y$ and all positive integers $i$ [4, p. 124].

Serre proved that a domain $Y$ in $\mathbb{C}^{n}$ is Stein if and only if $H^{i}\left(Y, \mathcal{O}_{Y}\right)=0$ for all $i>0$ [17], where $\mathcal{O}_{Y}$ is the analytic structure sheaf of $Y$. Laufer generalized Serre's

Received January 30, 2021; Accepted August 8, 2021.

Communicated by Min Ru.

2020 Mathematics Subject Classification. 32E10, 14A15, 14C20.

Key words and phrases. active germ, analytic hypersurface, analytic structure sheaf, Cěch cohomology, Stein space. 
result to Stein manifolds of dimension $m$ (see [4, pp. 159-160] or [10]). In algebraic geometry, Neeman showed that a quasi-compact Zariski open subset $Y$ of an affine scheme $X=\operatorname{Spec} A$ (with singularities) is affine if and only if $H^{i}\left(Y, \mathcal{O}_{Y}\right)=0$ for all $i>0$ [14, where $\mathcal{O}_{Y}$ is the algebraic structure sheaf of $Y$ and $A$ may not be noetherian.

In [10], Laufer also proved: If $Y$ is an $n$-dimensional Riemann domain over a Stein manifold such that $\mathcal{O}_{Y}$ separates points, and for all $i>0, H^{i}\left(Y, \mathcal{O}_{Y}\right)$ is a finite dimensional complex vector space, then $Y$ is a Stein manifold. A dimension $n$ Stein manifold can be biholomorphicall mapped onto a closed complex submanifold of $\mathbb{C}^{2 n+1}[7$, Ch. 5]. Laufer's proof heavily relies on the local coordinates of a complex manifold which cannot be applied to a singular Stein space.

For an open subset of a Stein space with singularities, we use complex algebraic geometry approach to avoid dealing with singularities directly and show the following result.

Theorem 1.1. If $Y$ is an open subset of a reduced Stein space $X$ of dimension $n$, then $Y$ is Stein if and only if $H^{i}\left(Y, \mathcal{O}_{Y}\right)$ is a finite dimensional complex vector space for every $i>0$.

A complex space $X$ is locally of finite dimension but globally its dimension may not be finite. If a nonempty complex space $X$ is irreducible, then there is a nonnegative integer $n \geq 0$ such that $\operatorname{dim}_{x} X=n$ for all $x \in X[5, \mathrm{p} .106]$ and $n$ is the dimension of $X$. If $X$ has infinitely many irreducible components, then the dimension of $X: \operatorname{dim} X=\sup _{x \in X} \operatorname{dim}_{x} X$ 5. p. 94] may not be finite and there are connected complex spaces with dimension $\infty 8$, p. 190]. Without the finite dimension condition, we have

Theorem 1.2. Let $Y$ be an open subset of a reduced Stein space $X$ with arbitrary dimension and singularities. Then $Y$ is Stein if and only if $H^{i}\left(Y, \mathcal{O}_{Y}\right)$ is a finite dimensional vector space over $\mathbb{C}$ for all $i>0$.

The idea of proof of Theorem 1.2 is the following 1 First, by Sard and Remmert's theorems, we can construct countably many holomorphic functions $f_{1}, f_{2}, \ldots$ such that each $f_{i}$ defines a (smooth) hypersurface $F_{i}$ with disconnected components, $G_{i}=F_{i} \cap Y$ is an open subset in $F_{i}$ and $1, f_{1}, f_{2}, \ldots$ are linearly independent (see Lemmas 2.9, 2.11, and 3.4). Here every $G_{i}$ is Stein, using the fact that it has disconnected components, MayerVietoris sequence and Theorem 1.1 (Lemma 3.7). Then we can show that for all $i>0$, $H^{i}\left(Y, \mathcal{O}_{Y}\right)=0$ by Lemma 3.7 and the dimension counting method of vector spaces due to

\footnotetext{
${ }^{1}$ The author was informed by an anonymous referee that Theorem 1.1 was proved by Vâjâitu for complex spaces (non-reduced) of dimension $n$ in 2010 and by modifying this proof and not using mathematical induction, the results in 21 hold for complex spaces with arbitrary dimension. The key idea in 21 to prove holomorphic convexity is to generalize an estimate of Fornæss and Narasimhan and use Wiegmann's construction to get a proper surjective morphism from a hypersurface to an $n$-dimensional Stein space.
} 
Goodman and Hartshorne [3] (see Lemmas 2.13 and 3.8). Finally, by Nagel's theorem on finitely generated property of the ring of holomorphic functions on $Y$ [12], we show that $Y$ is holomorphically convex (see Theorems 2.3 and 3.9 .

In [22 24], we investigated a question raised by J.-P. Serre [17]: Let $Y$ be a complex manifold with $H^{i}\left(Y, \Omega_{Y}^{j}\right)=0$ for all $j \geq 0$ and $i>0$ (where $\Omega_{Y}^{j}$ is the sheaf of holomorphic $j$-forms), then what is $Y$ ? Is $Y$ Stein? If $Y$ is an algebraic manifold (i.e., an irreducible nonsingular algebraic variety defined over $\mathbb{C}$ ) and $\Omega_{Y}^{j}$ is the sheaf of regular $j$-forms, we know that $Y$ is not an affine variety in general. In fact, if the dimension of $Y$ is $d$ and $X$ is a smooth projective variety containing $Y$, then $X$ may have $d-j$ algebraically independent nonconstant rational functions which are regular on $Y$, where $j=1,3, \ldots, d-2, d$ if $d$ is odd or $j=0,2, \ldots, d-2, d$ if $d$ is even. But the Steinness question is still open except for the trivial case when the dimension is one. By Theorem 1.1, we have

Corollary 1.3. If $Y$ is a nonsingular open subset of a Stein space $X$ with dimension $n$ such that $H^{i}\left(Y, \Omega_{Y}^{j}\right)=0$ for all $j \geq 0$ and $i>0$, then $Y$ is Stein.

Simha proved that an open subset of a normal Stein surface obtained by removing a closed analytic subspace of pure codimension one is a Stein surface [18]. This result does not hold for higher dimensional complex spaces with singularities (see an example in Section 3). For a normal Stein space, we have

Theorem 1.4. Let $Y$ be an open subset of a normal Stein space $X$ such that the complement $X-Y$ is a closed analytic subspace of $X$.

(1) If $X-Y$ is the support of an effective $\mathbb{Q}$-Cartier divisor, then $Y$ is Stein.

(2) If $X-Y$ is of pure codimension 1 and does not contain any singular points of $X$, then $Y$ is Stein.

In order to prove Theorem 1.2 in Section 3 , we first prove Theorem 1.1 in Section 2 by algebraic geometry approach. In Section 2, we also prove Theorem 2.3 for an open subset in a Stein space with arbitrary dimension and several lemmas which will be used in the proof of Theorem 1.2 in Section 3.

\section{Preparations}

A ring $R$ is local if it has exactly one maximal ideal $\mathcal{M}$. Every stalk $\mathcal{O}_{x}$ of the structure sheaf $\mathcal{O}_{X}$ of a complex space $X$ is a local ring: the maximal ideal $\mathcal{M}_{x} \subset \mathcal{O}_{x}$ consists of all germs at $x$ which can be represented in a neighborhood of $x$ by a holomorphic function. In fact, $\mathcal{O}_{x}$ is a local $\mathbb{C}$-algebra: the composition

$$
\phi: \mathbb{C} \cdot 1 \rightarrow \mathcal{O}_{x} \rightarrow \mathcal{O}_{x} / \mathcal{M}_{x}
$$


is an isomorphism of fields (see [5, pp. 5-7] or [8, p. 66, p. 97]).

Let $\mathcal{C}_{X}$ be the sheaf of germs of complex valued continuous functions on a Hausdorff topological space $X$. Then $\mathcal{C}_{X}$ is a local $\mathbb{C}$-algebra [5, p. 5]. Since the stalk

$$
\mathcal{O}_{x}=\mathbb{C} \oplus \mathcal{M}_{x}
$$

every germ $f_{x} \in \mathcal{O}_{x}$ can be uniquely written in the form

$$
f_{x}=c_{x}+m_{x},
$$

where $c_{x}$ is the complex value of $f_{x}$ at $x$ and $m_{x} \in \mathcal{M}_{x}$ [5, p. 8]. For a holomorphic function $f$ on an open subset $U$ of $X$, define a function $[f]: U \rightarrow \mathbb{C}$ by $[f](x)=c_{x}$ for $x \in U$. Then $f$ induces a continuous function $[f] \in \mathcal{C}_{X}(U)[5$, p. 9].

We need a theorem of Nagel [12].

Let $Y$ be a topological space, and let $\mathcal{A}$ be a sheaf of local $\mathbb{C}$-algebras on $Y$. We assume:

(a) For every $y \in Y$, the maximal ideal of the stalk $\mathcal{A}_{y}$ is $\mathcal{M}_{y}$, and the composition

$$
\phi: \mathbb{C} \cdot 1 \rightarrow \mathcal{A}_{y} \rightarrow \mathcal{A}_{y} / \mathcal{M}_{y}
$$

is an isomorphism.

(b) For every global section $f \in \Gamma(Y, \mathcal{A})$, the associated complex valued function $[f]$ is continuous, where $[f](y)$ is the residue class of the germ of $f$ at $y$ in $\mathcal{A}_{y} / \mathcal{M}_{y}$.

(c) For all $i>0, H^{i}\left(Y, \mathcal{O}_{Y}\right)=0$.

Lemma 2.1 (Nagel). Let $\mathcal{A}$ be a sheaf of local $\mathbb{C}$-algebras on $Y$ such that the above three conditions are satisfied. Suppose that $I$ is an ideal in $\Gamma(Y, \mathcal{A})$, and that there is a finite subset $\left\{f_{1}, f_{2}, \ldots, f_{m}\right\} \subset I$, so that for every $y \in Y$, there is an $f_{j}$ such that $f_{j}(y) \neq 0$. Then $I=\left(f_{1}, f_{2}, \ldots, f_{m}\right)=\Gamma(Y, \mathcal{A})$.

Lemma 2.2. Let $Y$ be an open subset of a Stein space $X$ such that $H^{i}\left(Y, \mathcal{O}_{Y}\right)=0$ for all $i>0$. If $h \in H^{0}\left(Y, \mathcal{O}_{Y}\right)$ is not a zero divisor of the stalk $\mathcal{O}_{y}$ at every point $y \in Y$, then $H^{i}\left(Z, \mathcal{O}_{Z}\right)=0$ for all $i>0$, where $Z=\{y \in Y, h(y)=0\}$ is the hypersurface defined by the holomorphic function $h$.

Proof. If $h$ is a unit in $H^{0}\left(Y, \mathcal{O}_{Y}\right)$, then $h$ does not vanish on $Y$ and $Z$ is an empty set. We assume that $h$ is not a unit on $Y$. The multiplication by $h$ defines an injective map from $\mathcal{O}_{Y}$ to itself. $Z$ is a hypersurface of pure codimension 1 on $Y[5$, p. 100] and we have a short exact sequence

$$
0 \longrightarrow \mathcal{O}_{Y} \longrightarrow \mathcal{O}_{Y} \longrightarrow \mathcal{O}_{Y} / h \mathcal{O}_{Y} \cong \mathcal{O}_{Z} \longrightarrow 0
$$


where the first map is defined by the non-zero divisor (a holomorphic function) $h$ on $X$. Since $H^{i}\left(Y, \mathcal{O}_{Y}\right)=0$ for all $i>0$, the corresponding long exact sequence gives

$$
0 \longrightarrow H^{0}\left(Y, \mathcal{O}_{Y}\right) \longrightarrow H^{0}\left(Y, \mathcal{O}_{Y}\right) \longrightarrow H^{0}\left(Y, \mathcal{O}_{Z}\right) \longrightarrow 0
$$

and $H^{i}\left(Z, \mathcal{O}_{Z}\right)=0$.

A holomorphic map $f: X \rightarrow X^{\prime}$ is proper if for every compact subset $K \in X^{\prime}$, the inverse image $f^{-1}(K) \subset X$ is a compact subset in $X$. Remmert's Proper Mapping Theorem states that for any proper holomorphic map $f: X \rightarrow X^{\prime}$ between complex spaces, the image $f(X)$ is an analytic subset of $X^{\prime}$ [5, p. 213].

In Theorem 2.3 , the dimension of the Stein space $X$ is arbitrary.

Theorem 2.3. If $Y$ is an open subset of a Stein space $X$ such that every accumulation point $P_{0} \in X-Y$ of a discrete sequence in $Y$ is the only common zero of finitely many holomorphic functions $f_{1}, \ldots, f_{m}$ on $X$, then $Y$ is Stein if and only if $H^{i}\left(Y, \mathcal{O}_{Y}\right)=0$ for all $i>0$.

Proof. If $Y$ is Stein, then for any coherent analytic sheaf $\mathcal{F}$ on $Y$ and all $i>0$, by Theorem $\mathrm{B}, H^{i}(Y, \mathcal{F})=0$. By Oka's theorem, the structure sheaf $\mathcal{O}_{Y}$ is coherent [5, p. 60] so $H^{i}\left(Y, \mathcal{O}_{Y}\right)=0$. We only need to show that if $H^{i}\left(Y, \mathcal{O}_{Y}\right)=0$ for all $i>0$, then $Y$ is Stein. $Y$ is holomorphically separable since holomorphic functions on $X$ separate points on the open subset $Y$. We will show that $Y$ is holomorphically convex.

Let $S=\left\{P_{1}, P_{2}, \ldots, P_{k}, \ldots\right\}$ be a discrete sequence in $Y$. If $S$ has no accumulation points in $X$, then there is a holomorphic function $f$ on $X$ such that $f$ is not bounded on $S$. We are done. We may assume that $S$ has an accumulation point $P_{0} \in X-Y$. Since there are finitely many holomorphic functions $f_{1}, \ldots, f_{m}$ on $X$ such that $P_{0} \notin Y$ is their only common zero, for every point $y \in Y$, at least one $f_{j}$ does not vanish at $y$. By Lemma 2.1, $f_{1}, f_{2}, \ldots, f_{m}$ generate the $\operatorname{ring} H^{0}\left(Y, \mathcal{O}_{Y}\right)=\Gamma\left(Y, \mathcal{O}_{Y}\right)$. Particularly, there are $g_{1}, g_{2}, \ldots, g_{m} \in H^{0}\left(Y, \mathcal{O}_{Y}\right)$ such that

$$
f_{1} g_{1}+f_{2} g_{2}+\cdots+f_{m} g_{m}=1
$$

on $Y$

Every holomorphic function $f_{i}$ is continuous on $X$ and $f_{i}\left(P_{0}\right)=0, i=1,2, \ldots, m$, so its limit at $P_{0} \in X-Y$ is 0 . By the equation, at least one $g_{j}$ has limit infinity at $P_{0}$. This implies that $g_{j}$ is not bounded on the discrete sequence $S$ since $P_{0} \in X-Y$ is an accumulation point of $S \subset Y$.

We show that $Y$ is holomorphically convex so it is Stein.

Lemma 2.4. Let $Y$ be an open subset of a Stein space $X$ with dimension $n$ such that $H^{i}\left(Y, \mathcal{O}_{Y}\right)=0$ for all $i>0$. Then $Y$ is holomorhically convex. 
Proof. Since $X$ is a Stein space of dimension $n$, there is a one-to-one, proper holomorphic map from $X$ to $\mathbb{C}^{2 n+1} 13$.

Let $S=\left\{P_{1}, P_{2}, \ldots, P_{k}, \ldots\right\}$ be a discrete sequence in $Y$. As in the proof of Theorem 2.3, we may assume that $S$ has an accumulation point $P_{0} \in X-Y$. By Narasimhan's theorem, let $\psi: X \rightarrow \mathbb{C}^{2 n+1}$ be a one-to-one, proper holomorphic map which is regular at every uniformizable point 13. By Remmert's Proper Mapping Theorem, $\psi(X)$ is a closed subspace of $\mathbb{C}^{2 n+1}$. By affine algebraic geometry, there are $m$ polynomials $f_{1}, f_{2}, \ldots, f_{m}$ in $\mathbb{C}^{2 n+1}$ such that the point $\psi\left(P_{0}\right)$ in $\psi(X)$ (not in $\psi(Y)$ ) is the only point in the zero set

$$
\left\{x \in \psi(X), f_{1}(x)=f_{2}(x)=\cdots=f_{m}(x)=0\right\} .
$$

Pull these polynomials back to $X$ by the proper injective holomorphic map $\psi$, we receive $m$ holomorphic functions (still denoted by $f_{i}$ for simplicity) $f_{1}, f_{2}, \ldots, f_{m}$ in $X$ such that their only common zero is the point $P_{0} \in X-Y$. So for every point $y \in Y$, at least one $f_{j}$ does not vanish at $y$. By Lemma 2.1, $f_{1}, f_{2}, \ldots, f_{m}$ generate $\Gamma\left(Y, \mathcal{O}_{Y}\right)$ and the rest of the proof is the same as proof of Theorem 2.3 .

We show that $Y$ is holomorphically convex so it is Stein.

By Lemma 2.4, we have

Theorem 2.5. If $Y$ is an open subset of a Stein space $X$ of dimension n, then $Y$ is Stein if and only if $H^{i}\left(Y, \mathcal{O}_{Y}\right)=0$ for all $i>0$.

Definition 2.6. (1) If $I$ is an ideal of a ring $R$, then the set

$$
\sqrt{I}=\left\{r \in R, r^{j} \in I, j \in \mathbb{N}\right\}
$$

is an ideal of $R$ called the radical of $I$ in $R$.

(2) An element $r \in R$ is an nilpotent element if there is a positive integer $n$ such that $r^{n}=0$.

(3) The radical $N=\sqrt{0}$ is called the nilradical of $R$.

(4) The ideal $I$ in a commutative ring $R$ is reduced if for $r \in R$, there is an integer $m \in \mathbb{N}, r^{m} \in I$, then $r \in I$.

Definition 2.7. The radical sheaf $\mathcal{N}=\sqrt{0}$ of the zero ideal in the structure sheaf $\mathcal{O}_{X}$ of a complex space $X$ is called the nilradical of $\mathcal{O}_{X}$.

By Definition 2.7, the stalk $\mathcal{N}_{x}$ is the ideal of all nilpotent germs in the stalk $\mathcal{O}_{x}$. For a complex space $X$, the nilradical $\mathcal{N}$ is a coherent ideal sheaf of $\mathcal{O}_{X}[5$, p. 86]. A complex space $X$ is reduced at a point $x_{0} \in X$ if the stalk $\mathcal{O}_{x_{0}}$ is reduced: $\mathcal{O}_{x_{0}}$ has no nilpotent 
elements. $X$ is reduced if for all points $x \in X$, all stalks $\mathcal{O}_{x}$ are reduced rings, i.e., if $f_{x} \in \mathcal{O}_{x}$ and $f_{x}^{m}=0$ for some $m \in \mathbb{N}$ ( $m$ relies on the point $x$ and the function $f$ ), then $f_{x}=0[8$, p. 151].

Definition 2.8. A germ $f_{x} \in \mathcal{O}_{x}$ at a point $x$ in a complex space $X$ is active if for every $g_{x} \in \mathcal{O}_{x}$, with $f_{x} g_{x} \in \mathcal{N}_{x}$, we have $g_{x} \in \mathcal{N}_{x}$.

The set of points of a complex space $X$ where $X$ is not reduced is an analytic subset of $X$ [5 p. 88]. A holomorphic function $f$ on $X$ is active at a point $x \in X$ if there is an open neighborhood $U$ of $x$ such that $f$ does not vanish at every irreducible component of $X$ in $U$ [5, p. 98].

Lemma 2.9. Let $\left\{1, f_{1}, f_{2}, \ldots, f_{m}, \ldots\right\} \subset V$ be linearly independent in a vector space $V$ over $\mathbb{C}$. Then for any constant $a_{i} \in \mathbb{C},\left\{1, f_{1}-a_{1}, f_{2}-a_{2}, \ldots, f_{m}-a_{m}, \ldots\right\}$ is also linearly independent in $V$.

Proof. For any $m \in \mathbb{N}$, let $c_{i} \in \mathbb{C}, i=0,1,2, \ldots, m$ and

$$
c_{0}+c_{1}\left(f_{1}-a_{1}\right)+c_{2}\left(f_{2}-a_{2}\right)+\cdots+c_{m}\left(f_{m}-a_{m}\right)=0 .
$$

Then

$$
\left(c_{0}-c_{1} a_{1}-c_{2} a_{2}-\cdots-c_{m} a_{m}\right)+c_{1} f_{1}+c_{2} f_{2}+\cdots+c_{m} f_{m}=0 .
$$

Since $1, f_{1}, f_{2}, \ldots, f_{m}$ are linearly independent, we have

$$
c_{0}-c_{1} a_{1}-c_{2} a_{2}-\cdots-c_{m} a_{m}=c_{1}=c_{2}=\cdots=c_{m}=0 .
$$

So $c_{0}=c_{1}=c_{2}=\cdots=c_{m}=0$ and $1, f_{1}-a_{1}, f_{2}-a_{2}, \ldots, f_{m}-a_{m}$ are linearly independent. Similarly, we can show that any finite subset of $\left\{1, f_{1}-a_{1}, f_{2}-a_{2}, \ldots, f_{m}-a_{m}, \ldots\right\}$ is linearly independent. Therefore, $\left\{1, f_{1}-a_{1}, f_{2}-a_{2}, \ldots, f_{m}-a_{m}, \ldots\right\}$ is linearly independent.

Lemma 2.10. If $X$ is a Stein space of dimension at least 1 , then the dimension $h^{0}\left(X, \mathcal{O}_{X}\right)$ of the vector space $H^{0}\left(X, \mathcal{O}_{X}\right)$ over $\mathbb{C}$ is not finite.

Proof. We will construct infinitely many holomorphic functions on $X$ which are linearly independent.

Let $C$ be an irreducible analytic curve in $X$ and $\mathcal{I}_{C}$ be its ideal sheaf in $X$ such that $C \cap Y$ is an open subset of $C$ and contains smooth points in $X$. Then $\mathcal{I}_{C}$ is coherent analytic sheaf on $X[5$, p. 84]. We have a short exact sequence

$$
0 \rightarrow \mathcal{I}_{C} \rightarrow \mathcal{O}_{X} \rightarrow \mathcal{O}_{X} / \mathcal{I}_{C}=\mathcal{O}_{C} \rightarrow 0
$$

Since $X$ is Stein, $H^{1}\left(X, \mathcal{I}_{C}\right)=0$ and we have a surjective map $H^{0}\left(X, \mathcal{O}_{X}\right) \rightarrow H^{0}\left(C, \mathcal{O}_{C}\right)$. 
Let $P_{0}$ be a smooth point of the curve $C$ and $X$ and $z$ be the local coordinate at $P_{0}$ on $C$. Now $C$ is a Stein curve so there is a nonconstant holomorphic function $f$ on $C$ such that $f(z)=z+z^{2} g(z)$ near $P_{0}$ [4, p. 151], where $g(z)$ is holomorphic near $P_{0}$. Then $1, f, f^{2}, \ldots, f^{m}, \ldots$ are holomorphic functions on $C$ and linearly independent over C. Since $h^{0}\left(C, \mathcal{O}_{C}\right)=\infty$, we have $h^{0}\left(X, \mathcal{O}_{X}\right)=\infty$.

Lemma 2.11. If $X$ is a reduced Stein space of arbitrary dimension, then there are infinitely many holomorphic functions $1, f_{1}-a_{1}, f_{2}-a_{2}, \ldots, f_{m}-a_{m}, \ldots$ on $X$ such that they are linearly independent in $H^{0}\left(Y, \mathcal{O}_{Y}\right)$ and each of $f_{1}-a_{1}, f_{2}-a_{2}, \ldots, f_{m}-a_{m}, \ldots$ defines a reduced hypersurface on $Y$.

Proof. By Lemma 2.10, there are holomorphic functions $1, f_{1}, f_{2}, \ldots, f_{m}, \ldots$ on $X$ such that they are linearly independent in the vector space $H^{0}\left(X, \mathcal{O}_{X}\right)$ over $\mathbb{C}$. Each function $f_{i}$ gives a nonconstant holomorphic map from $X$ to $\mathbb{C}$. By open mapping theorem, if $f_{i}$ is not a constant near a point $p \in X$, then the map $f_{i}: X \rightarrow \mathbb{C}$ is open near $p$ [5, p. 109]. So the image $f_{i}(X)$ contains an open subset $V$ of $\mathbb{C}$. By the Sard type theorem, there is a countable subset $B \subset \mathbb{C}$ such that for every point $a_{i} \in \mathbb{C}-B$, the fiber (hypersurface) $X_{a_{i}}=f_{i}^{-1}\left(a_{i}\right)$ is reduced 11 . We may choose suitable $a_{i}$ such that each $f_{i}-a_{i}$ defines a reduced hypersurface in $Y$.

By the construction in the proof of Lemma 2.10, we may choose these holomorphic functions so that they are linearly independent on an irreducible curve $C$ (i.e., linearly independent in $\left.H^{0}\left(C, \mathcal{O}_{C}\right)\right)$ in $X$ such that $C \cap Y$ is an open subset of $C$, then they are linearly independent in $H^{0}\left(Y, \mathcal{O}_{Y}\right)$. This is because if we have

$$
c_{0}+c_{1} f_{1}+c_{2} f_{2}+\cdots+c_{m} f_{m}=0
$$

on $Y$, then $c_{0}+c_{1} f_{1}+c_{2} f_{2}+\cdots+c_{m} f_{m}=0$ on the curve $C \cap Y$. By the Identity Theorem 5 , p. 170], the equation holds on the irreducible curve $C$. But these functions are linearly independent on $C$, we have $c_{0}=c_{1}=\cdots=c_{m}=0$ and they are linearly independent on $Y$. By Lemma 2.9, $\left\{1, f_{1}-a_{1}, f_{2}-a_{2}, \ldots, f_{m}-a_{m}, \ldots\right\}$ is linearly independent in $H^{0}\left(Y, \mathcal{O}_{Y}\right)$.

Lemma 2.12. Let $Y$ be an open subset of a Stein space $X$ of arbitrary dimension such that $H^{i}\left(Y, \mathcal{O}_{Y}\right)$ is a finite dimensional vector space over $\mathbb{C}$ for all $i>0$, then the dimension $h^{i}\left(Z, \mathcal{O}_{Z}\right)<\infty$ for every hypersurface $Z$ defined by a holomorphic function $h$ on $Y$ which is not a zero divisor of $\mathcal{O}_{y}$ at every point $y \in Y$.

Proof. Since $h$ is not a zero divisor on $Y$, we have a short exact sequence

$$
0 \longrightarrow \mathcal{O}_{Y} \longrightarrow \mathcal{O}_{Y} \longrightarrow \mathcal{O}_{Y} / h \mathcal{O}_{Y} \cong \mathcal{O}_{Z} \longrightarrow 0
$$


where the first map is defined by $h$. The corresponding long exact sequence gives

$$
\begin{aligned}
0 \longrightarrow H^{0}\left(Y, \mathcal{O}_{Y}\right) \longrightarrow H^{0}\left(Y, \mathcal{O}_{Y}\right) \longrightarrow H^{0}\left(Z, \mathcal{O}_{Z}\right) \longrightarrow H^{1}\left(Y, \mathcal{O}_{Y}\right) \longrightarrow H^{1}\left(Y, \mathcal{O}_{Y}\right) \\
\quad \stackrel{\alpha}{\longrightarrow} H^{1}\left(Z, \mathcal{O}_{Z}\right) \stackrel{\beta}{\longrightarrow} H^{2}\left(Y, \mathcal{O}_{Y}\right) \longrightarrow H^{2}\left(Y, \mathcal{O}_{Y}\right) \longrightarrow H^{2}\left(Z, \mathcal{O}_{Z}\right) \longrightarrow \cdots
\end{aligned}
$$

The sequence is exact at $H^{1}\left(Z, \mathcal{O}_{Z}\right)$ so the relationship between the image of $\alpha$ and kernel of $\beta$ is

$$
\operatorname{im}(\alpha)=\alpha\left(H^{1}\left(Y, \mathcal{O}_{Y}\right)\right)=\operatorname{ker}(\beta) \subset H^{1}\left(Z, \mathcal{O}_{Z}\right)
$$

and

$$
\operatorname{dim}_{\mathbb{C}} \operatorname{ker}(\beta)=\operatorname{dim}_{\mathbb{C}} \operatorname{im}(\alpha) \leq h^{1}\left(Y, \mathcal{O}_{Y}\right)<\infty
$$

$\beta$ is a homomorphism from the vector space $H^{1}\left(Z, \mathcal{O}_{Z}\right)$ to the vector space $H^{2}\left(Y, \mathcal{O}_{Y}\right) 16$, pp. 627-629], so the image vector space $\operatorname{im}(\beta)$ is a subspace of $H^{2}\left(Y, \mathcal{O}_{Y}\right)$. This implies

$$
\operatorname{dim}_{\mathbb{C}} \operatorname{im}(\beta) \leq h^{2}\left(Y, \mathcal{O}_{Y}\right)<\infty
$$

By the rank theorem in linear algebra, these two inequalities give $h^{1}\left(Z, \mathcal{O}_{Z}\right)<\infty$. Using the fact that the sequence is exact at $H^{i}\left(Z, \mathcal{O}_{Z}\right)$ and $h^{i}\left(Y, \mathcal{O}_{Y}\right)<\infty$ for all $i>0$, $h^{i}\left(Z, \mathcal{O}_{Z}\right)<\infty$ can be similarly proved.

Lemma 2.13. Let $Y$ be an open subset of a reduced Stein space $X$ of dimension $n$ such that $H^{i}\left(Y, \mathcal{O}_{Y}\right)$ is a finite dimensional vector space over $\mathbb{C}$ for all $i>0$, then $H^{i}\left(Y, \mathcal{O}_{Y}\right)=0$.

Proof. For every holomorphic function $f \in H^{0}\left(Y, \mathcal{O}_{Y}\right)$, the multiplication by $f$ induces a homomorphism:

$$
f^{* i}: H^{i}\left(Y, \mathcal{O}_{Y}\right) \longrightarrow H^{i}\left(Y, \mathcal{O}_{Y}\right)
$$

and the map $f \rightarrow f^{* i}$ is a $\mathbb{C}$-homomorphism (see [3] or [16, pp. 627-629])

$$
H^{0}\left(Y, \mathcal{O}_{Y}\right) \longrightarrow \operatorname{End}_{\mathbb{C}}\left(H^{i}\left(Y, \mathcal{O}_{Y}\right)\right)
$$

where $\operatorname{End}_{\mathbb{C}}(V)$ is the set of all vector homomorphisms (linear transformations) from a vector space $V$ over $\mathbb{C}$ to itself. Since $H^{i}\left(Y, \mathcal{O}_{Y}\right)$ is a finite dimensional vector space over $\mathbb{C}$ for all $i>0, \operatorname{End}_{\mathbb{C}}\left(H^{i}\left(Y, \mathcal{O}_{Y}\right)\right)$ is also a finite dimensional vector space over $\mathbb{C}$ for all $i>0$.

By Lemma 2.11, there are infinitely many holomorphic functions $1, f_{1}, f_{2}, \ldots, f_{m}, \ldots$ on $X$ such that they are linearly independent and each of them defines a reduced hypersurface on $X$. Each $f_{j}$ defines a homomorphism $f_{j}^{* i}$ from the vector space $H^{i}\left(Y, \mathcal{O}_{Y}\right)$ to itself. But $\operatorname{End}_{\mathbb{C}}\left(H^{i}\left(Y, \mathcal{O}_{Y}\right)\right)$ is a finite dimensional vector space over $\mathbb{C}$ for all $i>0$, so for each $i$, there is an $f_{l_{i}} \subset\left\{f_{1}, f_{2}, \ldots, f_{m}, \ldots\right\}$ such that it induces a zero map from $H^{i}\left(Y, \mathcal{O}_{Y}\right)$ to itself. By the choice of the functions, $f_{l_{i}}$ defines a reduced hypersurface $Z_{l_{i}}$. 
We will use mathematical induction on the dimension of $X$ to show that for all $i>0$, $H^{i}\left(Y, \mathcal{O}_{Y}\right)=0$.

If $X$ is a Stein curve, then $Y$ is an open subset of $X$ and for any coherent sheaf $\mathcal{F}$ on $Y$ and all $i>0, H^{i}(Y, \mathcal{F})=0[19]$ so $H^{i}\left(Y, \mathcal{O}_{Y}\right)=0$ for all $i>0$.

If $X$ is a Stein surface and $h^{i}\left(Y, \mathcal{O}_{Y}\right)<\infty$ for all $i>0$, then the hypersurface $Z$ defined by a holomorphic function (non-zero divisor) $f$ on $Y$ is Stein 19 so $H^{1}\left(Z, \mathcal{O}_{Z}\right)=0$ and $H^{2}\left(Z, \mathcal{O}_{Z}\right)=0$. Since $Y$ is an open surface, $H^{i}\left(Y, \mathcal{O}_{Y}\right)=0$ for all $i>1[19$.

By the above long exact sequence,

$$
f^{* 1}: H^{1}\left(Y, \mathcal{O}_{Y}\right) \rightarrow H^{1}\left(Y, \mathcal{O}_{Y}\right)
$$

is surjective. Now for all $j \in \mathbb{N}$, we have infinitely many surjective group homomorphisms $f_{j}^{* 1}$ of a finite dimensional vector space $H^{1}\left(Y, \mathcal{O}_{Y}\right)$

$$
f_{j}^{* 1}: H^{1}\left(Y, \mathcal{O}_{Y}\right) \rightarrow H^{1}\left(Y, \mathcal{O}_{Y}\right)
$$

induced by each $f_{j} \in\left\{f_{1}, f_{2}, \ldots, f_{m}, \ldots\right\}$. Because $\operatorname{End}_{\mathbb{C}}\left(H^{i}\left(Y, \mathcal{O}_{Y}\right)\right)$ is a finite dimensional vector space over $\mathbb{C}$ for all $i>0$, by counting the dimensions of vector spaces, we see that there is a $k \in \mathbb{N}$ such that $f_{k}^{* 1}=0\left[3\right.$. But $f_{k}^{* 1}$ is a surjective map from $H^{1}\left(Y, \mathcal{O}_{Y}\right)$ to itself. We see $H^{1}\left(Y, \mathcal{O}_{Y}\right)=0$.

We receive $H^{i}\left(Y, \mathcal{O}_{Y}\right)=0$ for all $i>0$.

By mathematical induction, we may assume that if dimension of $X$ is $n-1$, and $h^{i}\left(Y, \mathcal{O}_{Y}\right)<\infty$ for all $i>0$, then $H^{i}\left(Y, \mathcal{O}_{Y}\right)=0$ for all $i>0$.

Let $X$ be a Stein space of dimension $n$ in the lemma and $h^{i}\left(Y, \mathcal{O}_{Y}\right)<\infty$ for all $i>0$. By Lemma 2.12, any reduced hypersurface $Z$ defined by a holomorphic function satisfies $h^{i}\left(Z, \mathcal{O}_{Z}\right)<\infty$ for all $i>0$. By inductive assumption, $H^{i}\left(Z, \mathcal{O}_{Z}\right)=0$ for all $i>0$. Using the long exact sequence, for every $f_{j} \in\left\{f_{1}, f_{2}, \ldots, f_{m}, \ldots\right\}$ we have infinitely many surjective maps

$$
f_{j}^{* 1}: H^{1}\left(Y, \mathcal{O}_{Y}\right) \rightarrow H^{1}\left(Y, \mathcal{O}_{Y}\right)
$$

and isomorphisms

$$
f_{j}^{* i}: H^{i}\left(Y, \mathcal{O}_{Y}\right) \rightarrow H^{i}\left(Y, \mathcal{O}_{Y}\right)
$$

for $i>1$. By counting the dimensions of the vector spaces, we see that for all $i>0$, $H^{i}\left(Y, \mathcal{O}_{Y}\right)=0$ because $\operatorname{End}_{\mathbb{C}}\left(H^{i}\left(Y, \mathcal{O}_{Y}\right)\right)$ is a finite dimensional vector space over $\mathbb{C}$ for all $i>0$.

Lemma 2.14. Let $Y$ be an open subset of a reduced Stein space $X$ of dimension $n$ such that $H^{i}\left(Y, \mathcal{O}_{Y}\right)$ is a finite dimensional vector space over $\mathbb{C}$ for all $i>0$, then the ring of holomorphic functions on $Y$ is finitely generated: there are holomorphic functions $f_{1}, f_{2}, \ldots, f_{m}$ on $Y$ such that they generate $H^{0}\left(Y, \mathcal{O}_{Y}\right)$. 
Proof. By Lemma 2.13, $H^{i}\left(Y, \mathcal{O}_{Y}\right)=0$ for all $i>0$. By Lemma 2.1 and proof of Lemma 2.4 $H^{0}\left(Y, \mathcal{O}_{Y}\right)$ is generated by finitely many holomorphic functions $f_{1}, f_{2}, \ldots, f_{m}$ on $Y$.

Theorem 2.15. Let $Y$ be an open subset of a reduced Stein space $X$ with dimension $n$. Then $Y$ is Stein if and only if $H^{i}\left(Y, \mathcal{O}_{Y}\right)$ is a finite dimensional complex vector space for every $i>0$.

Proof. By Lemma 2.13, if $H^{i}\left(Y, \mathcal{O}_{Y}\right)$ is a finite dimensional complex vector space for every $i>0$, then $H^{i}\left(Y, \mathcal{O}_{Y}\right)=0$. By Theorem 2.5, $Y$ is Stein.

In Section 3, we will prove that Theorem 2.15 holds if the dimension of $X$ is not finite.

\section{Spaces with arbitrary dimension}

We will first prove Theorem 1.2 in this section.

By Remmert's Proper Mapping Theorem (see [5, p. 213] or [15, Satz 23]), if $f: X \rightarrow Y$ is a proper holomorphic map, then the image of any analytic set in $X$ is again analytic in $Y$. If $f$ is not proper, this is not true.

Definition 3.1. A subset $A$ of a complex space $X$ is said to be analytically meagre if $A \subset \bigcup_{i \in \mathbb{N}} A_{i}$, where each $A_{i}$ is a locally analytic subset of $X$ with codimension at least 1 .

An analytically meagre subset of a curve is a countable set [11. If $f$ is not proper, Remmert proved (see [11] or [15, Satz 20]).

Lemma 3.2 (Remmert). If $f: X \rightarrow Y$ is a holomorphic map between complex spaces and $Z$ is an analytic subset of $X$, then $f(Z)$ is a countable union of locally analytic subsets of $Y$. In particular, if the interior of $f(Z)$ is empty, then $f(Z)$ is analytically meagre.

An analytic subset $Z$ in a complex space $X$ is always nowhere dense in $X$ if $Z$ is at least 1 codimensional in $X$ and $Z$ contains interior points of $X$ if $Z$ contains an irreducible component of $X$ [5, pp. 102-103].

Lemma 3.3 (Sard). If $X$ is a complex manilold and $f: X \rightarrow \mathbb{C}$ is a holomorphic function, then there exists a countable subset $A \subset \mathbb{C}$ such that for each $c \in \mathbb{C}-A$, the fiber $X_{c}=$ $f^{-1}(c)$ is a manifold.

The following construction is inspired by Remmert and Sard's theorems.

Lemma 3.4. Let $Y$ be an open subset of a reduced Stein space $X$, then there is a holomorphic function $h$ on $X$ such that for any $a \in \mathbb{C}-A$, the hypersurface defined by $h-a$ on $X$ is a complex manifold $H=H_{1} \cup H_{2} \cup \cdots$ of codimension 1 in $X$ and for all $i \neq j$, $H_{i} \cap H_{j}=\emptyset$, where $A$ is a countable subset in $\mathbb{C}$. 
Proof. Since $X$ is reduced, the singular locus $X_{\text {sing }}$ of $X$ is a nowhere dense analytic subset of $X$ (i.e., for every open subset $U$ in $X, U \cap X_{\text {sing }}$ is not dense in $U$ ) such that the local dimension at $x: \operatorname{dim}_{x} X_{\text {sing }}<\operatorname{dim}_{x} X$ [5, p. 117]. Let $X=X_{1} \cup X_{2} \cup \cdots$ be the decomposition of $X$ into irreducible components [4, p. 19]. The singular locus $X_{\text {sing }}$ consists of all intersection points of $X_{i} \cap X_{j}, i \neq j$ and the singular points of each component $X_{i}[5$, p. 117].

First, we claim that there is a holomorphic function $h$ on $X$ such that it is not a constant on $Y$ and $h\left(X_{\text {sing }}\right)$ is nowhere dense in $\mathbb{C}$. In fact, let $C$ be a holomorphic curve in $X$ such that $C \cap Y$ is an open subset of $C$ and $C \cap X_{\text {sing }}$ is empty (that is, $C$ contains

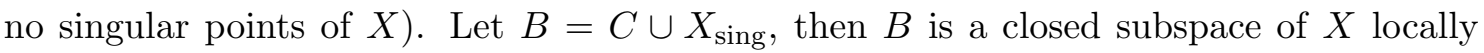
defined by holomorphic functions $\left[5\right.$, p. 15]. Let $\mathcal{I}_{B}$ be the ideal sheaf of $B$, then we have a short exact sequence

$$
0 \longrightarrow \mathcal{I}_{B} \longrightarrow \mathcal{O}_{X} \longrightarrow \mathcal{O}_{C \cup X_{\text {sing }}} \longrightarrow 0
$$

Since $X$ is Stein and $\mathcal{I}_{B}$ is a coherent ideal sheaf, $H^{1}\left(X, \mathcal{I}_{B}\right)=0$. We have a surjective map $H^{0}\left(X, \mathcal{O}_{X}\right) \rightarrow H^{0}\left(B, \mathcal{O}_{C \cup X_{\text {sing }}}\right)$. By the fact that $C$ and $X_{\text {sing }}$ are disconnected, we may construct a holomorphic function $h$ on $B$ such that $h$ is not a constant on $C$ and $h\left(X_{\text {sing }}\right)$ is nowhere dense in $\mathbb{C}$ (for example, we may choose $h$ such that it is a constant on every connected component of $\left.X_{\text {sing }}\right)$.

By Lemma 3.2, $h\left(X_{\text {sing }}\right)=A_{1}$ is a countable union of locally analytic subsets so is a countable subset of $\mathbb{C}$. For any $a \in \mathbb{C}-A_{1}$, the fiber $X_{a}=h^{-1}(a)$ has no intersection points with the singular locus $X_{\text {sing }}$ of $X$. But the hypersurface $X_{a} \subset X-X_{\text {sing may }}$ have singular points as a closed subspace of $X$. Now the restriction $h: X-X_{\text {sing }} \rightarrow \mathbb{C}$ is a holomorphic function on the complex manifold $X-X_{\text {sing }}$. By Sard's Theorem, there exists a countable subset $A_{2} \subset \mathbb{C}$ such that for each $c \in \mathbb{C}-A_{2}$, the fiber

$$
X_{c} \cap\left(X-X_{\text {sing }}\right)=h^{-1}(c) \cap\left(X-X_{\text {sing }}\right) \subset X-X_{\text {sing }}
$$

is a manifold. $h$ may be a constant at some irreducible component of $X$. Since $X$ has at most a countably many irreducible components [4, p. 19], there is a countable subset $A_{3} \subset \mathbb{C}$ such that for every $a \in \mathbb{C}-A_{3}$, the fiber $X_{a}=h^{-1}(a)$ is of pure codimension 1 in $X$. Let $A=A_{1} \cup A_{2} \cup A_{3}$, then $A$ is the union of three countable subsets so is a countable subset of $\mathbb{C}$. For all $a \in \mathbb{C}-A$, the fiber $X_{a}=h^{-1}(a)$ is of pure codimension 1 in $X$, smooth and can be decomposed into the union of disjoint complex manifolds. Therefore $H=X_{a}=H_{1} \cup H_{2} \cup \cdots$ is a smooth hypersurface in $X$, each $H_{i}$ is irreducible and for all $i \neq j, H_{i} \cap H_{j}=\emptyset$.

Lemma 3.5. Let $Y$ be an open subset of a reduced Stein space $X$ such that $H^{i}\left(Y, \mathcal{O}_{Y}\right)$ is a finite dimensional vector space over $\mathbb{C}$ for all $i>0$. In above lemma, for every irreducible 
component $H_{j}$ of hypersurface $H$, let $Z=Y \cap H$ and $Z_{j}=Y \cap H_{j}$, then $Z_{j}$ is an open subset in $H_{j}$ and

$$
h^{i}\left(Z_{j}, \mathcal{O}_{Z}\right)<\infty
$$

Proof. We may assume that $X$ contains no isolated points since $X$ is Stein then every connected component of $X$ is Stein [4, p. 125]. Let $Z=H \cap Y=Z_{1} \cup Z_{2} \cup \cdots$, where $Z_{i}=Y \cap H_{i}$ is either empty or an open subset in $H_{i}$ (the subspace topology on $H_{i}$ is induced from the topology on $X$ since $Y$ is open in $X$ and $H_{i}$ is a closed subspace of $X$ ).

If $Z_{j}$ is an empty set or a set of points, then the inequality is true. We may assume that the dimension of $Z_{j}$ is at least one. Let $Z=Z_{j} \cup Z_{j}^{\prime}$, where $Z_{j}^{\prime}=Z-Z_{j}$ is the complement of $Z_{j}$ in $Z$. By the construction in Lemma 3.4, $Z_{j} \cap Z_{j}^{\prime}$ is empty and by Mayer-Vietoris sequence [1, p. 30], we have

$$
\begin{aligned}
& 0 \longrightarrow H^{0}\left(Z, \mathcal{O}_{Z}\right) \longrightarrow H^{0}\left(Z_{j}, \mathcal{O}_{Z}\right) \oplus H^{0}\left(Z_{j}^{\prime}, \mathcal{O}_{Z}\right) \longrightarrow H^{0}\left(Z_{j} \cap Z_{j}^{\prime}, \mathcal{O}_{Z}\right) \\
& \longrightarrow H^{1}\left(Z, \mathcal{O}_{Z}\right) \longrightarrow H^{1}\left(Z_{j}, \mathcal{O}_{Z}\right) \oplus H^{1}\left(Z_{j}^{\prime}, \mathcal{O}_{Z}\right) \longrightarrow H^{1}\left(Z_{j} \cap Z_{j}^{\prime}, \mathcal{O}_{Z}\right) \\
& \longrightarrow H^{2}\left(Z, \mathcal{O}_{Z}\right) \longrightarrow H^{2}\left(Z_{j}, \mathcal{O}_{Z}\right) \oplus H^{2}\left(Z_{j}^{\prime}, \mathcal{O}_{Z}\right) \longrightarrow H^{2}\left(Z_{j} \cap Z_{j}^{\prime}, \mathcal{O}_{Z}\right) \longrightarrow \cdots
\end{aligned}
$$

Since $Z_{j} \cap Z_{j}^{\prime}=\emptyset, H^{i}\left(Z_{j} \cap Z_{j}^{\prime}, \mathcal{O}_{Z}\right)=0$ for all $i \geq 0$, we have

$$
H^{i}\left(Z, \mathcal{O}_{Z}\right) \cong H^{i}\left(Z_{j}, \mathcal{O}_{Z}\right) \oplus H^{i}\left(Z_{j}^{\prime}, \mathcal{O}_{Z}\right)
$$

By Lemma 2.12, for all $i>0$,

$$
h^{i}\left(Z, \mathcal{O}_{Z}\right)<\infty
$$

so $h^{i}\left(Z_{j}, \mathcal{O}_{Z}\right)<\infty$, and $h^{i}\left(Z_{j}^{\prime}, \mathcal{O}_{Z}\right)<\infty$.

Lemma 3.6. In above lemma, for every irreducible component $H_{j}$ of hypersurface $H$ such that $Z_{j}=Y \cap H_{j} \neq \emptyset, Z_{j}$ is a Stein subset in $H_{j}$.

Proof. The hyersurface $H$ in $X$ is Stein [4, p. 125]. Since $H=H_{1} \cup H_{2} \cup \cdots$ and for all $i \neq j, H_{i} \cap H_{j}=\emptyset$, every irreducible (thus connected) component $H_{i}$ is Stein [4, p. 125]. For each irreducible component $H_{i}$, its dimension is a constant [5, p. 169] even though the dimension of $H$ may not be finite. By Lemma 3.5 and Theorem 2.15, the nonempty open subset $Z_{j}$ in $H_{j}$ is a Stein open subset in $H_{j}$.

Lemma 3.7. In above lemmas, the hypersurface $Z=H \cap Y$ in the open subset $Y$ is holomorphically convex therefore is Stein.

Proof. Let $S=\left\{P_{1}, P_{2}, \ldots, P_{k}, \ldots\right\}$ be a discrete sequence in $Z=Z_{1} \cup Z_{2} \cup \cdots$, where $Z_{i}=H_{i} \cap Y$. As in the proof of Theorem 2.3. we may assume that it has an accumulation point $P_{0}$ in $X$. 
If there is an irreducible hypersurface $Z_{j} \subset H_{j} \subset H$ such that $Z_{j} \cap S \subset H_{j} \cap S$ contains infinitely many points of $S$, then there is a holomorphic function $f$ on $Z_{j}$ such that $f$ is not bounded on $Z_{j} \cap S$. By Mayer-Vietoris sequence,

$$
H^{i}\left(Z, \mathcal{O}_{Z}\right) \cong H^{i}\left(Z_{j}, \mathcal{O}_{Z}\right) \oplus H^{i}\left(Z_{j}^{\prime}, \mathcal{O}_{Z}\right)
$$

we can extend $f$ to the complement $Z_{j}^{\prime}$ of $Z_{j}$ in $Z$ by zero since $Z j$ and $Z_{j}^{\prime}$ are disconnected. In this way, we receive a holomorphic function $f$ on $Z$ such that it is not bounded on $S$.

Now we assume that every nonempty component $Z_{i}$ only contains finitely many points of $S$ and $S$ has an accumulation point $P_{0}$ in $X$. Choose a subsequence $\left\{P_{n_{i}}\right\}_{i=1}^{\infty}$ in $S$ such that $P_{0} \in X-Z$ is its limit point. Let the holomorphic function $h$ define the hypersurface $H$ in $X$. Since $h\left(P_{i}\right)=0$ for all $i$, we have $h\left(P_{0}\right)=0$. This implies that $P_{0}$ is a point on some irreducible component $H_{k}$ of $H$. By Lasker-Noether Decomposition Theorem, there is an open subset $U \ni P_{0}$ in $X$ such that in $U, H$ has only finitely many components: $H \cap U=H_{i_{1}} \cup H_{i_{2}} \cup \cdots \cup H_{i_{m}}$ [5, pp. 78-79]. But each irreducible component in $H \cap U$ contains only finitely many points of $S, P_{0}$ cannot be an accumulation point of $S$. The contradiction implies that if $S=\left\{P_{1}, P_{2}, \ldots, P_{k}, \ldots\right\} \subset Z$ has an accumulation point in $X$, then there is a component $H_{j}$ such that $H_{j} \cap S$ is not a finite set. By the above proof, we show that there is a holomorphic function $f$ on $Z$ such that it is not bounded on $S$. So the hypersurface $Z$ in the open subset $Y$ is holomorphically convex.

Lemma 3.8. Let $Y$ be an open subset of a reduced Stein space $X$ such that $H^{i}\left(Y, \mathcal{O}_{Y}\right)$ is a finite dimensional vector space over $\mathbb{C}$ for all $i>0$, Then

$$
H^{i}\left(Y, \mathcal{O}_{Y}\right)=0
$$

Proof. By the construction in Lemmas 2.11 and 3.4, let $f_{1}, f_{2}, \ldots, f_{m}, \ldots$ be holomorphic functions on $X$ such that $1, f_{1}, f_{2}, \ldots, f_{m}, \ldots$ are linearly independent in $H^{0}\left(Y, \mathcal{O}_{Y}\right)$ and for every $i$, each image $f_{i}\left(X_{\text {sing }}\right)$ in $\mathbb{C}$ is nowhere dense. By Lemma 3.4, choose $a_{j} \in \mathbb{C}$ such that each fiber $X_{a_{j}}=f_{i}^{-1}\left(a_{j}\right)$ defines a pure codimension 1 complex manifold $X_{a_{j}}$ in $X$. By Lemma 2.9, $1, f_{1}-a_{1}, f_{2}-a_{2}, \ldots, f_{m}-a_{m}, \ldots$ are linearly independent in $H^{0}\left(Y, \mathcal{O}_{Y}\right)$. By Lemmas 3.4 3.7, each $Y_{a_{j}}=Y \cap X_{a_{j}}$ is a smooth Stein hypersurface on $Y$, so $h^{i}\left(Y_{a_{j}}, \mathcal{O}_{Y_{a_{j}}}\right)=0$ for all $i>0$. Using the idea of the proof of Lemma 2.13 , multiplicating by each $f_{j}-a_{j}$ from $\mathcal{O}_{Y}$ to itself for all $j \in \mathbb{N}$, we have infinitely many surjective $\mathbb{C}$-homomorphisms $\left(f_{j}-a_{j}\right)^{* 1}$ of a finite dimensional vector space $H^{1}\left(Y, \mathcal{O}_{Y}\right)$

$$
\left(f_{j}-a_{j}\right)^{* 1}: H^{1}\left(Y, \mathcal{O}_{Y}\right) \rightarrow H^{1}\left(Y, \mathcal{O}_{Y}\right)
$$

and infinitely many $\mathbb{C}$-isomorphisms

$$
\left(f_{j}-a_{j}\right)^{* i}: H^{i}\left(Y, \mathcal{O}_{Y}\right) \rightarrow H^{i}\left(Y, \mathcal{O}_{Y}\right)
$$


which are induced by each $f_{j}-a_{j} \in\left\{f_{1}-a_{1}, f_{2}-a_{2}, \ldots, f_{m}-a_{m}, \ldots\right\}$ for $i>1[3]$. Comparing the dimensions of vector spaces, for all $i>0$, we have $H^{i}\left(Y, \mathcal{O}_{Y}\right)=0$.

Theorem 3.9. Let $Y$ be an open subset of a reduced Stein space $X$ such that $H^{i}\left(Y, \mathcal{O}_{Y}\right)$ is a finite dimensional vector space over $\mathbb{C}$ for all $i>0$. Then $Y$ is holomorphically convex therefore is Stein.

Proof. Let $S=\left\{P_{1}, P_{2}, \ldots, P_{k}, \ldots\right\}$ be a discrete sequence in $Y$ and $P_{0}$ be its accumulation in $X$. Since $X$ is Stein, $X$ is holomorphically spreadable, that is, there exist finitely many holomorphic functions $f_{1}, f_{2}, \ldots, f_{m}$ on $X$ such that $P_{0}$ is an isolated point in the zero set $A=\left\{x \in X, f_{1}(x)=f_{2}(x)=\cdots=f_{m}(x)=0\right\}$ [8, pp. 293-294]. We can write $A=B \cup\left\{P_{0}\right\}$ then $B \cap\left\{P_{0}\right\}$ is an empty set. Let $\mathcal{I}_{A}$ be the ideal generated by $f_{1}, f_{2}, \ldots, f_{m}$ in $X$, then we have a short exact sequence

$$
0 \longrightarrow \mathcal{I}_{A} \longrightarrow \mathcal{O}_{X} \longrightarrow \mathcal{O}_{X} / \mathcal{I}_{A}=\mathcal{O}_{A} \longrightarrow 0
$$

The ideal sheaf $\mathcal{I}_{A}$ is coherent on the Stein space $X$ [5, p. 84]. The long exact sequence and $H^{1}\left(X, \mathcal{I}_{A}\right)=0$ give

$$
0 \longrightarrow H^{0}\left(X, \mathcal{I}_{A}\right) \longrightarrow H^{0}\left(X, \mathcal{O}_{X}\right) \longrightarrow H^{0}\left(A, \mathcal{O}_{A}\right) \longrightarrow 0
$$

Let $f_{m+1} \in H^{0}\left(A, \mathcal{O}_{A}\right)$ such that $f_{m+1}\left(P_{0}\right)=0$ and $B \cap\left\{x \in X, f_{m+1}(x)=0\right\}=\emptyset$. Then there is a holomorphic function (still denoted by $f_{m+1}$ ) on $X$ such that it vanishes at $P_{0}$ and does not vanish at every point of $B$.

Now $f_{1}, f_{2}, \ldots, f_{m}, f_{m+1}$ are holomorphic on $X$ and have a unique common zero $P_{0}$ on $X$. They have no common zeros on $Y$. By Lemma 3.8, for all $i>0, H^{i}\left(Y, \mathcal{O}_{Y}\right)=0$. By Theorem 2.3 , $Y$ is Stein.

We have proved

Theorem 3.10. Let $Y$ be an open subset of a reduced Stein space $X$ with arbitrary dimension and singularities. Then $Y$ is Stein if and only if $H^{i}\left(Y, \mathcal{O}_{Y}\right)$ is a finite dimensional vector space over $\mathbb{C}$ for all $i>0$.

Next we will prove Theorem 1.4

Definition 3.11. A Weil divisor on a reduced complex space $X$ is a locally finite linear combination with integral coefficients of irreducible reduced analytic subspaces of codimension 1 in $X$ such that every subspace is not contained in the singular locus of $X$.

The set of all Weil divisors form an abelian group. If $D$ is a Weil divisor, then we can write $D=\sum_{i=1}^{\infty} n_{i} D_{i}$, where $n_{i} \in \mathbb{Z}$ and each $D_{i}$ is an irreducible reduced analytic 
subspace of codimension 1 in $X$ which is not contained in the singular locus of $X$ (see [2], 4, pp. 139-140], [6, pp. 130-143], or [20, pp. 35-36]).

The support of a Weil divisor $D$ is the union of all closed subspaces $D_{i}$ such that $n_{i} \neq 0$. $D$ is an effective divisor, written $D>0$, if every coefficient $n_{i} \geq 0$ and $D$ is not a zero divisor. Two Weil divisors $D \geq D^{\prime}$ if $D-D^{\prime} \geq 0$, i.e., $D-D^{\prime}$ is an effective divisor or a zero divisor in the space. When every coefficient $n_{i}=1, D=\sum D_{i}$ is called a reduced divisor.

A reduced point $x \in X$ is a normal point of $X$ if the stalk $\mathcal{O}_{x}$ is integrally closed in its quotient ring. A reduced complex space is normal if every point in the space is a normal point [5, p. 8]. If $X$ is a compact normal reduced complex space, then a Weil divisor $D$ is a finite sum on $X: D=\sum_{i=1}^{N} n_{i} D_{i}[20$, p. 35].

If $X$ is normal, then the singular locus of $X$ is a closed subspace of codimension at least 2 in $X$ [5, p. 128]. A Weil divisor is well-defined as a linear combination of irreducible codimension one closed subspaces on a normal complex space $X$.

A Cartier divisor $D$ on a complex space $X$ is a global section of the sheaf $\mathcal{M}_{X}^{*} / \mathcal{O}_{X}^{*}$, where $\mathcal{M}_{X}^{*}$ is the sheaf of germs of not identically vanishing meromorphic functions on $X$ and $\mathcal{O}_{X}^{*}$ is the sheaf of germs of nowhere vanishing holomorphic functions on $X$. A Cartier divisor $D$ on a complex space $X$ can be described by an appropriate open cover $\left\{U_{i}\right\}_{i \in I}$ of $X$ and a collection of meromorphic functions $f_{i}$ on $U_{i}, i \in I$ such that on $U_{i} \cap U_{j} \neq \emptyset, \frac{f_{i}}{f_{j}}$ and $\frac{f_{j}}{f_{i}}$ are holomorphic (see [4, p. 138] or [20, p. 30]). D is an effective Cartier divisor, written $D>0$, if every $f_{i}$ is a holomorphic function and at least one of them has zeros [20, p. 31].

Every Cartier divisor on a normal reduced complex space $X$ defines a Weil divisor and if $X$ is nonsingular, then every Weil divisor is Cartier, i.e., locally it is defined by one equation. But if $X$ is not a complex manifold, then the Weil divisor $D$ is not a Cartier divisor in general, i.e., it is not locally defined by one equation [20, p. 36].

A Weil divisor $D$ is $\mathbb{Q}$-Cartier if there is an $n \in \mathbb{N}$ such that $n D$ is a Cartier divisor, i.e., $n D$ is locally defined by one equation.

Example 3.12. Let $X \in \mathbb{C}^{4}$ be a quadric threefold defined by

$$
X=\left\{z=\left(z_{1}, z_{2}, z_{3}, z_{4}\right) \in \mathbb{A}_{k}^{4}, p(z)=z_{1}^{2}+z_{2}^{2}+z_{3}^{2}+z_{4}^{2}=0\right\}
$$

The structure sheaf

$$
\mathcal{O}_{X}=\mathcal{O}_{\mathbb{C}^{4}} / p(z) \mathcal{O}_{\mathbb{C}^{4}}
$$

$X$ is a normal Stein variety with a unique isolated singularity at 0 . Let $H$ be a hypersurface through 0 defined by

$$
H=\left\{z=\left(z_{1}, z_{2}, z_{3}, z_{4}\right) \in X, z_{1}=i z_{2}, z_{3}=i z_{4}\right\}
$$


$H$ cannot be defined by a single holomorphic function and $X-H$ is not Stein [4, p. 130].

Example 3.12 shows that the open subset $Y=X-A$ in a normal Stein space $X$ obtained by removing a pure codimension 1 subspace $A$ of $X$ is not Stein in general if the dimension of $X$ is at least 3 . We give a sufficient condition:

Theorem 3.13. If $Y$ is an open subset of a normal Stein space $X$ such that the complement $X-Y$ is a closed analytic subspace of $X$ and the support of an effective $\mathbb{Q}$-Cartier divisor, then $Y$ is Stein.

Proof. Let $D^{\prime}$ be the effective $\mathbb{Q}$-Cartier divisor with support $X-Y$ on $X$. Then there is an $n \in \mathbb{N}$ such that $D=n D^{\prime}$ is an effective Cartier divisor with support $X-Y$ on $X$ [20, pp. 36-38].

Let $\left\{U_{i}\right\}_{i \in I}$ be a Stein open cover of $X$ and let $f_{i}$ be the holomorphic function on $U_{i}$ defining $\left.D\right|_{U_{i}}$. Then for every point $x \in U_{i}$, the stalk of the invertible sheaf (coherent) $\mathcal{O}_{X}(D)$ is defined by $[20$, p. 30]

$$
\mathcal{O}_{X}(D)_{x}=\frac{1}{f_{i}} \mathcal{O}_{x} \cong \mathcal{O}_{x}
$$

Let $S=\left\{P_{1}, P_{2}, \ldots\right\} \subset Y$ be a discrete sequence on $Y$ with an accumulation point $P_{0} \in(X-Y) \cap U_{i}$ for some $i \in I$. Since $\mathcal{O}_{X}(D)$ is a coherent sheaf on $X$, by Cartan's Theorem A [4, p. 124], the module of global sections $H^{0}\left(X, \mathcal{O}_{X}(D)\right)$ generates every stalk $\mathcal{O}_{X}(D)_{x}$. There is a meromorphic function $f \in H^{0}\left(X, \mathcal{O}_{X}(D)\right)$ (holomorphic on $Y$ with poles in $X-Y$ ) and a local holomorphic function $g \in \mathcal{O}_{P_{0}}$ such that near $P_{0}$ [4, p. 129],

$$
\frac{1}{f_{i}}=f g \text {. }
$$

Now $f_{i}$ is a holomorphic function on $U_{i} \cap Y$ and vanishes at $P_{0}$. So $f\left(f_{i} g\right)=1$ near $P_{0}$ in $Y$. From $f_{i}\left(P_{0}\right)=0$, we see that $f$ is not bounded near $P_{0}$ on the sequence $S$ in $Y$. We show that $Y$ is holomorphically convex therefore is Stein.

Remark 3.14. A Stein open subset of an algebraic affine variety is not an algebraic affine variety in general. For example, let $X=\mathbb{C}^{n}$, let $Z$ be the closed analytic subvariety of $X$ defined by $f(z)=\sin z_{1}$, where $\left(z_{1}, z_{2}, \ldots, z_{n}\right)$ are coordinates in $\mathbb{C}^{n}$. Then $Y=X-Z$ is Stein but not an algebraic variety.

Surprisingly, Neeman constructed an example: there is a scheme $U$ of finite type over $\mathbb{C}$ such that $U$ is a Zariski open subset of an affine scheme and the associated analytic complex space $U^{\prime}$ of $U$ is a Stein space, but $U$ is not an affine scheme [14.

Theorem 3.15. If $Y$ is an open subset of a Stein space $X$ such that the complement $X-Y$ is a closed analytic subspace of $X$ with pure codimension 1 and $X-Y$ does not contain any singular points of $X$, then $Y$ is Stein. 
Proof. By Reduction Theorem [4, p. 154], $X$ is Stein if and only if its reduction is Stein. The normalization of a reduced complex space is a finite surjective holomorphic map [4. p. 22]. So a complex space is Stein if and only if its normalization is Stein 8, p. 313, Prop. 73.1]. The normalization $\widetilde{X}$ of $X$ is a disjoint union of irreducible components and it is Stein if and only if every irreducible component is Stein [8, p. 308, Cor. 71.14]. Therefore we may assume that $X$ is an irreducible normal (reduced) Stein space.

Let $X_{\text {sing }}$ be the set of singular points of $X$. Then $X_{\text {sing }}$ is of codimension at least 2 in $X$ [5, p. 128], and $X-Y \subset X-X_{\text {sing }}$ is a closed subspace of pure codimension 1 in the complex manifold $X-X_{\text {sing. }}$. Since every point in $X-Y$ is smooth in $X,(X-Y) \cap X_{\text {sing }}$ is an empty set. So $X-Y$ is support of an effective Cartier divisor $D$ in the complex manifold $X-X_{\text {sing }}[20$, p. 36].

Let $\left\{\left(U_{i}, f_{i}\right)\right\}_{i \in I}$ be a representive of $D$ in the complex manifold $X-X_{\text {sing, }}$, where $\left\{U_{i}\right\}_{i \in I}$ is a Stein open cover of the complex manifold $X-X_{\text {sing }}$, each $f_{i}$ is a holomorphic function on $U_{i}$, at least one $f_{i}$ has zeros, and $f_{i} / f_{j}$ is a holomorphic function on $U_{i} \cap U_{j}$ for all $i, j \in I$.

Let $\left\{V_{j}\right\}_{j \in J}$ be a Stein open cover of $X_{\text {sing }}$ in $Y: X_{\text {sing }} \subset \cup_{j} V_{j} \subset Y$. On each $V_{j} \cap U_{i} \neq \emptyset$, $\left.f_{i}\right|_{V_{j} \cap U_{i}}$ is nowhere zero. In particular, on every $V_{j}-V_{j} \cap X_{\text {sing }}$, we have $[20$, p. 36, Thm. 4.13]

$$
\mathcal{O}_{V_{j}-V_{j} \cap X_{\text {sing }}}(D) \cong \mathcal{O}_{V_{j}-V_{j} \cap X_{\text {sing }}}
$$

Now the codimension of $V_{j} \cap X_{\text {sing }}$ is at least 2 in $V_{j}$, therefore the invertible sheaf $\mathcal{O}_{V_{j}-V_{j} \cap X_{\text {sing }}}(D)$ can be extended to $V_{j}$ uniquely [9]. This implies that we have an invertible sheaf $\mathcal{O}_{X}(D)$ on $X$, i.e., $D$ is an effective Cartier divisor on $X$ [6, p. 144, Prop. 6.13]. By Theorem 3.13, $Y$ is Stein.

Corollary 3.16. If $Y$ is an open subset of a Stein manifold $X$ such that the complement $X-Y$ is a closed subspace of $X$ with pure codimension 1 , then $Y$ is Stein.

\section{References}

[1] D. Arapura, Complex Algebraic Varieties and their Cohomology, Lecture Notes, 2003, https://www.math.purdue.edu/ arapura/preprints/sheaves.pdf.

[2] E. Artal Bartolo, J. Martín-Morales and J. Ortigas-Galindo, Cartier and Weil divisors on varieties with quotient singularities, Internat. J. Math. 25 (2014), no. 11, 1450100, 20 pp.

[3] J. Goodman and R. Hartshorne, Schemes with finite-dimensional cohomology groups, Amer. J. Math. 91 (1969), 258-266. 
[4] H. Grauert and R. Remmert, Theory of Stein Spaces, Grundlehren der Mathematischen Wissenschaften 236, Springer-Verlag, Berlin, 1979.

[5] _ Coherent Analytic Sheaves, Grundlehren der Mathematischen Wissenschaften 265, Springer-Verlag, Berlin, 1984.

[6] R. Hartshorne, Algebraic Geometry, Graduate Texts in Mathmatics 52, SpringerVerlag, New York, 1977.

[7] L. Hörmander, An Introduction to Complex Analysis in Several Variables, Second revised edition, North-Holland Mathematical Library 7, North-Holland Publishing Co., Amsterdam, 1973.

[8] L. Kaup and B. Kaup, Holomorphic Functions of Several Variables: An introduction to the fundamental theory, De Gruyter Studies in Mathematics 3, Walter de Gruyter \& Co., Berlin, 1983.

[9] J. Kollár, The structure of algebraic threefolds: An introduction to Mori's program, Bull. Amer. Math. Soc. (N.S.) 17 (1987), no. 2, 211-273.

[10] H. B. Laufer, On sheaf cohomology and envelopes of holomorphy, Ann. of Math. (2) 84 (1966), 102-118.

[11] M. Manaresi, Sard and Bertini type theorems for complex spaces, Ann. Mat. Pura Appl. (4) 131 (1982), 265-279.

[12] A. Nagel, Cohomology, maximal ideals, and point evaluations, Proc. Amer. Math. Soc. 42 (1974), 47-50.

[13] R. Narasimhan, Imbedding of holomorphically complete complex spaces, Amer. J. Math. 82 (1960), 917-934.

[14] A. Neeman, Steins, affines and Hilbert's fourteenth problem, Ann. of Math. (2) 127 (1988), no. 2, 229-244.

[15] R. Remmert, Holomorphe und meromorphe Abbildungen komplexer Räume, Math. Ann. 133 (1957), 328-370.

[16] O. Riemenschneider, Singular Points of Complex Analytic Surface: An introduction to the local analysis of complex analytic spaces, Chapter 19, Supplement: A short introduction to complex analytic spaces. Retrieved from https://www.math.uni-hamburg.de/home/riemenschneider/bookchap30.pdf 
[17] J.-P. Serre, Quelques problèmes globaux relatifs aux variétés de Stein, in: Colloque sur les fonctions de plusieurs variables, tenu à Bruxelles, 57-68, Georges Thone, Liège, Paris, 1953.

[18] R. R. Simha, On the complement of a curve on a Stein space of dimension two, Math. Z. 82 (1963), 63-66.

[19] Y.-t. Siu, Analytic sheaf cohomology groups of dimension $n$ of $n$-dimensional complex spaces, Trans. Amer. Math. Soc. 143 (1969), 77-94.

[20] K. Ueno, Classification Theory of Algebraic Varieties and Compact Complex Spaces, Lecture Notes in Mathematics 439, Springer-Verlag, Berlin, 1975.

[21] V. Vâjâitu, A cohomological Steinness criterion for holomorphically spreadable complex spaces, Czechoslovak Math. J. 60 (2010), no. 3, 655-667.

[22] J. Zhang, Threefolds with vanishing Hodge cohomology, Trans. Amer. Math. Soc. 357 (2005), no. 5, 1977-1994.

[23] _ On the existence of nontrivial 3-folds with vanishing Hodge cohomology, Michigan Math. J. 54 (2006), no. 2, 447-467.

[24] _ Complex manifolds with vanishing Hodge cohomology, Houston J. Math. 43 (2017), no. 3, 807-827.

Jing Zhang

1 University Parkway, Department of Mathematics, Division of Science, Mathematics and Technology, College of Arts and Sciences, Governors State University, University Park, IL 60484, USA

E-mail address: jzhang@govst.edu 